\title{
Simulation of Internal Crack Detection in Shape-Distorted Cast Billets Using Time-of-Flight Measurements of Longitudinal Ultrasonic Waves
}

\author{
Hideto Mitsui*, Tadashi Ebihara*, Koichi Mizutani and Naoto Wakatsuki \\ Graduate School of Systems and Information Engineering, University of Tsukuba, Tsukuba 305-8573, Japan
}

To detect cracks in shape distorted cast billets, we newly propose a method that is the use of ultrasonic computerized tomography (CT) in combination with measurements of time-of-flight (TOF) of longitudinal ultrasonic waves at multiple planes as a more robust reconstruction method. The TOF is measured by applying the transmission method, and the measured TOF is used to reconstruct the apparent sound velocity profile. Cracks are identified as a decrease in the apparent sound velocity, which is caused by an increase in the TOF after the diffraction of ultrasonic waves at the cracks. The combination makes it possible to remove the effects of shape distortion to be compensated by the TOF measurements at multiple planes. Therefore, cracks are clearly detected using this method. The detection ability of this method is evaluated by numerical simulations of a shape-distorted billet. The results of this study are validated with help of wave propagation simulations using the transmission line matrix (TLM) method. More specifically, the newly proposed method allowed us to detect cracks that could hardly be distinguished with the use of our previously proposed method owing to fluctuations of the apparent sound velocity caused by shape distortions. In addition, using the new method enables the detection of cracks located near the billet's surface whose dimensional change is as small as $5 \%$. From the results of this study, we believe that the newly proposed method could be useful for the crack detection in shape-distorted billets. [doi:10.2320/matertrans.I-M2011854]

(Received November 12, 2009; Accepted October 29, 2011; Published January 25, 2012)

Keywords: ultrasonic testing, time-of-flight, longitudinal waves, transmission method, reconstruction

\section{Introduction}

Billets produced by continuous casting are often subjected to excessive stresses during cooling and extrusion processes. ${ }^{1-3)}$ These shear and compressive stresses may cause cracks as well as shape distortions. Despite numerous improvements in the casting process, it can still be difficult to overcome some of these problems and produce billets entirely free from defects. ${ }^{4,5}$ Crack detection is therefore a prerequisite for maintaining the quality of the final products. Moreover, it is essential to maintain balance between the competing goals for increasing productivity and reducing shape distortions. ${ }^{6-8)}$ Then, productivity usually has priority because billets are primary products.

For the detection of cracks in billets, the ultrasonic pulse echo method has been extensively used in the past. ${ }^{9}{ }^{910}$ ) This method can be used to detect cracks by measuring echoes reflected from the cracks. However, the echoes may not be received if the grain size of the billet is so large that the input signals are attenuated during propagation. In other words, this method has not proved to be reliable for detecting cracks and it has not been put to practical use.

We have recently proposed an alternative method to detect cracks by measuring the time-of-flight (TOF) of longitudinal waves. ${ }^{11)}$ The TOF is measured by the transmission method because the intensity of the ultrasonic wave transmitted is larger than that of an echo pulse. An increase in TOF is attributed to the diffraction of ultrasonic waves at existing cracks. More specifically, cracks are detected as a decrease in the apparent sound velocity that is reconstructed on the basis of the TOF measurements. For the reconstruction of the apparent sound velocity profile, ultrasonic computerized tomography (CT) is employed. This method can detect cracks, whose lengths are longer than the wavelength of input

*Graduate Student, University of Tsukuba signal, in a billet only when its dimensions are held to designed values. However, shape distortions are also particularly harmful to crack detection. In particular, for the reconstruction of the apparent sound velocity profile, CT uses the lengths of those propagation paths in the billets. Therefore, shape distortions affect the reliability of this method for crack detection because the lengths of the propagation paths in shape-distorted billet vary with the degree of distortion.

In this paper, we propose new method that is the use of ultrasonic CT in combination with the measurements of the TOF at multiple planes for the reconstruction of the apparent sound velocity profiles. This combination makes it possible to remove the effects of shape distortions to be compensated so that cracks are more clearly detected in comparison to the previously proposed method, because the lengths of the propagation paths are not used. The detection ability of this method was evaluated by numerical simulations using the model of a shape-distorted billet. The ultrasonic wave propagation was estimated using the transmission-line matrix (TLM) method.

\section{Principles of Detection}

\subsection{Measurement of TOF}

To detect a crack, we used longitudinal ultrasonic waves as input waves. This choice was based on the fact that the first waves to be received are the longitudinal waves because they are the fastest elastic waves. In other words, mode conversions and shear and surface waves have no influence on measurement of the TOF of longitudinal waves. A chirp signal is the input signal transmitted and received according to the transmission method. The chirp signal is often used in cross-correlation analysis because it facilitates the detection of a peak even if the direct and reflected waves interfere with each other. ${ }^{12,13)}$ The TOF is defined as the elapsed time 


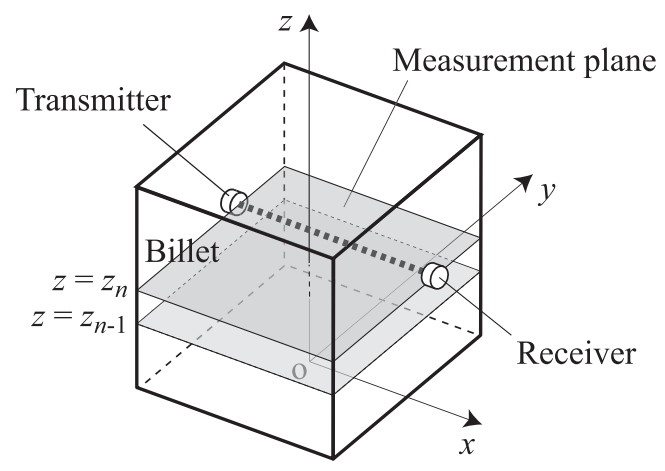

Fig. 1 Measurement system.

between the sending out of the ultrasonic wave and the first peak of the cross-correlation of input and received signals. ${ }^{14)}$ The direct wave is usually received in the absence of cracks on the sound propagation path. However, when a crack is located on the sound propagation path, the received wave is no longer the direct wave but the wave diffracted at the crack - at least in the case of an open crack. This means that the TOF of the diffracted wave is significantly longer than that of direct wave. Therefore, we can detect a crack by utilizing the increase in TOF that carries the necessary information for the existence of cracks in billets.

\subsection{Visualization of cracks}

Cracks are visualized using the apparent sound velocity profile, which is reconstructed on the basis of the measured TOF of the longitudinal waves. It should be noted that the apparent sound velocities of longitudinal waves are significantly lower at cracks than at crack-free areas because the TOF increases after their diffraction at the cracks. In other words, a decrease in the apparent sound velocity indicates the existence of cracks.

Figure 1 shows a schematic drawing of the measurement system. The origin of the rectangular coordinate system is considered to be at the center of the billet's bottom face. The measurement plane indicated as $z=z_{n}$ is parallel to the $x-y$ plane. Figure 2 shows a measurement plane of the shapedistorted billet being tested. The cross-sectional image of the measurement plane is produced by the reconstruction method. Measurements are made at multiple planes along the $z$-direction by moving both the transmitter and the receiver. We examined two different methods, which are the previously proposed method and the newly proposed method, for the reconstruction of the cross-sectional images.

The previously proposed method consists of the TOF measurements at just one plane. ${ }^{15)}$ An increase in the TOF due to diffraction is projected on the measurement plane $z_{n},{ }^{16)} p_{z_{n}}(X, \theta)$, as follows:

$$
p_{z_{n}}(X, \theta)=T_{z_{n}}(X, \theta)-L_{z_{n}}(X, \theta) / c
$$

where $T_{z_{n}}(X, \theta)$ denotes the TOF at point $(X, \theta)$ on the measurement plane $z_{n}, c$ denotes the sound velocity in the billet, and $L_{z_{n}}(X, \theta)$ denotes the length of the propagation path along the $Y$-direction passing through point $(X, \theta)$, which is calculated from information on the billet's dimensions if they are held to designed values. Therefore, this method may be difficult to detect the crack owing to the shape distortions.

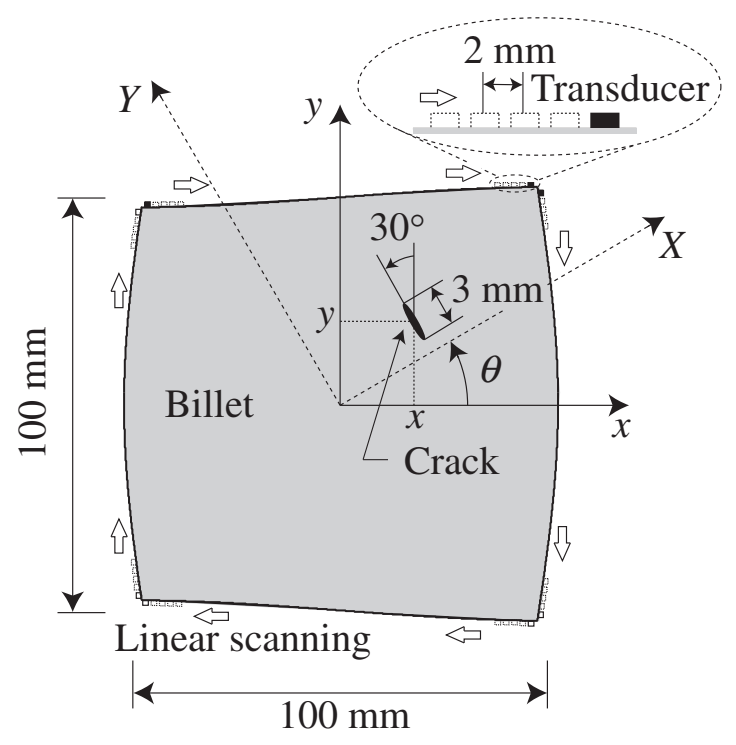

Fig. 2 Measurement plane of shape-distorted billet.

On the other hand, the newly proposed method includes the TOF measurements at multiple planes. An increase in the TOF, $p_{z_{n}}(X, \theta)$, of the longitudinal waves is expressed as follows:

$$
p_{z_{n}}(X, \theta)=T_{z_{n}}(X, \theta)-T_{z_{n-1}}(X, \theta) .
$$

The subscript $z_{n-1}$ denotes the plane located inversely next to the plane $z_{n}$. We suppose that the length of propagation path on the $z_{n}$ plane is almost equal to that on the $z_{n-1}$ plane, because despite the shape distortions, the billet remains continuous in shape. Therefore, with the newly proposed method, the effect of shape distortions is compensated through the calculation of the TOF difference between two adjacent planes rather than the length of propagation paths calculated accurately only in the case of billets without shape distortions.

The cross-sectional image $f(x, y)$ is reconstructed using the difference of the TOF, $p_{z_{n}}(X, \theta)$, by filtered backprojection. ${ }^{17,18)}$ The apparent sound velocity $c_{\mathrm{a}}(x, y)$ is given by the following expression:

$$
c_{\mathrm{a}}(x, y)=1 /\left(f(x, y)+c^{-1}\right) .
$$

\section{Verification by Numerical Simulations}

The detection ability of the newly proposed method was validated with help of wave propagation simulations using the TLM method. ${ }^{19,20)}$ Figure 2 shows the model billet under investigation that was made of steel and through which sound traveled at a velocity $c$ (approximately $5900 \mathrm{~m} \cdot \mathrm{s}^{-1}$ ). In the numerical simulations, we assumed that the size of each side of the billet, $S_{0}$, was $100 \mathrm{~mm}$. In addition, changes in the billet's dimensions, $d$, were defined as the ratio of the dimensional allowance to the target size, ${ }^{21)}$ which is given by the following expression:

$$
d=\max \left|S-S_{0}\right| / S_{0},
$$

where $S$ denotes the size of shape-distorted billet. The shape distortions were simply approximated by either a sine or a cosine curve, which are given by the following expressions: 


$$
\left\{\begin{array}{l}
y_{\text {upper }}(x)=d \times\left(S_{0} / 2\right) \times \sin \left(\pi x / S_{0}\right) \\
y_{\text {lower }}(x)=-d \times\left(S_{0} / 2\right) \times \sin \left(\pi x / S_{0}\right) \\
x_{\text {right }}(y)=d \times S_{0} \times\left\{\cos \left(\pi y / S_{0}\right)-1 / 2\right\} \\
x_{\text {left }}(y)=-d \times S_{0} \times\left\{\cos \left(\pi y / S_{0}\right)-1 / 2\right\}
\end{array},\right.
$$

where $y_{\text {upper }}$ and $y_{\text {lower }}$ denote distortions of the upper and lower sides of the steel billet and $x_{\text {right }}$ and $x_{\text {left }}$ denote those on the right and left sides, respectively. In eq. (5), the maximum and minimum size of shape-distorted billet correspond to $(1+d) S_{0}$ and $(1-d) S_{0}$, respectively. The crack was located at point $(x, y)$. The length of the crack was set to $3 \mathrm{~mm}$, which is almost equal to the wavelength of the input signal, and its direction was set at angles of $30^{\circ}$ with the $y$-axis. Transducers, namely, a transmitter and a receiver, were set on the billet, which were scanned linearly in the direction perpendicular to $z$-axis at short steps of $2 \mathrm{~mm}$ in order to cover the perimeter of the billet in sufficient detail. For this purpose, 50 measuring points were selected on each side of the billet. The diameter of the transducer was $2 \mathrm{~mm}$. The detection ability had been found to be declined according to the increase in the transducer diameter. Therefore, the transducer's diameter should be comparatively small. More specifically, a crack, whose length is greater than the wavelength, may be detected using a transducer of $2 \mathrm{~mm}$ in diameter. $^{22)}$ The input signal was a chirp signal whose frequency was increased from 1 to $3 \mathrm{MHz}$ in $5 \mu \mathrm{s}$. The wavelength of input signal ranged from 2 to $6 \mathrm{~mm}$.

First, we tested the detection ability of the previously proposed method, which is based on measurement of TOF from a single plane. Figure 3 shows the images of cracks obtained. The crack is located at $(x, y)=(20,10)(\mathrm{mm})$. Figures 3(a)-3(c) show the images for $d=0,2.5$, and 5\%, respectively. The dimensional change $d$ was assumed to be below $5 \%$ because that of actual manufactured billets is within $5 \%$. The gray scale indicates differences in the apparent sound velocity $c_{\mathrm{a}}$. Cracks were visualized in Figs. 3(a) and 3(b) as a decrease in the apparent sound velocity. The location of the visualized crack was estimated to be at point $(20,10)$. In addition, either an increase or a decrease in the apparent sound velocity was observed near the billet's surface as the dimensional change increases. This was due to either a decrease or an increase in the length of the sound propagation path owing to shape distortions. For example, in Fig. 3(c), the apparent sound velocity decreased down to $5638 \mathrm{~m} \cdot \mathrm{s}^{-1}$ near the point $(50,0)$, whereas at point $(20,10)$, where a crack was located, the apparent sound velocity was $5689 \mathrm{~m} \cdot \mathrm{s}^{-1}$. This means that the effect of shape distortions is stronger than that of cracks and affects the method's ability to detect cracks, as shown in Fig. 3(c).

Our next step was to test the ability of the newly proposed method to detect cracks using the TOF measurements at multiple planes. Figure 4 shows the simulation setup and reconstructed images of the apparent sound velocity. The dimensional change $d$ was fixed at 5\%. Figure 4(a) shows the setup of single crack. Figures 4(b) and 4(c) show images obtained with the previously proposed method and newly proposed method, respectively. For the numerical simulations, we assumed that the conditions on plane $z_{n}$ were the same as those in Fig. 2. It should be noted that in the absence of cracks, the measurement plane $z_{n-1}$ approximates the plane

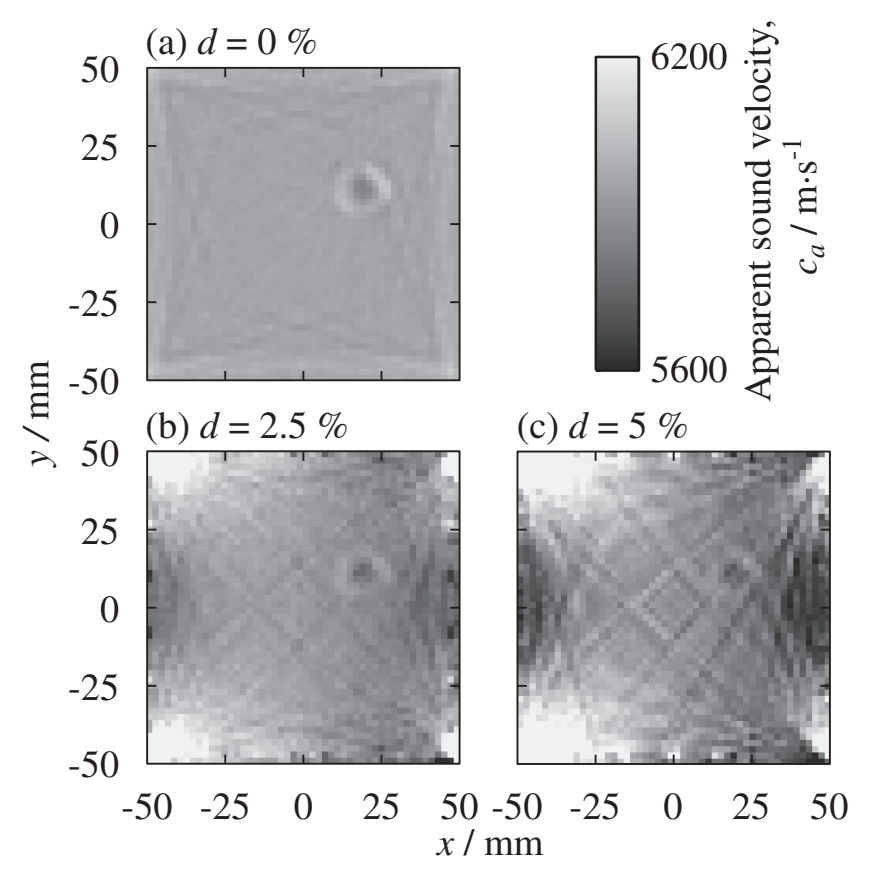

Fig. 3 Images obtained with previously proposed method: (a)-(c) show images for a dimensional change $d=0,2.5$, and $5 \%$, respectively.

$z_{n}$. From Fig. 4(c) which is obtained with the newly proposed method, the apparent sound velocity of the billet is estimated to be approximately $5900 \mathrm{~m} \cdot \mathrm{s}^{-1}$, which is equal to the velocity at which sound travels through the steel billet. A crack was identified by the apparent sound velocity that was significantly lower at the crack than in the area around it. In the ultrasonic CT image, the decrease in the apparent sound velocity, which is attributed to shape distortions, was compensated because in the newly proposed method, we used the TOF difference between two adjacent planes.

We also tested the crack detection of multiple cracks with the use of both methods. For example, three cracks can be identified in Fig. 4(d). For the simulations, we assumed that the conditions were the same as those for a single crack. The cracks were located at $(x, y)=(20,5),(20,20)$, and $(20,35)$ $(\mathrm{mm})$. Figures 4(e) and 4(f) show the images obtained with the previously proposed method and the newly proposed method, respectively. In Fig. 4(e), it is difficult to distinguish any crack from shape distortions. Multiple cracks cannot be observed as well as a single crack in Fig. 4(b). In Fig. 4(f), which shows images obtained with the newly proposed method, all three cracks can be identified prominently. However, the three cracks are different in the apparent sound velocity. The apparent sound velocity of the crack closer to the surface seems to be decreased.

Finally, we tested the detection ability of the two reconstruction methods using a model where a crack is located near the surface of the steel billet. Figure 5 shows the simulation setup and images of the crack. Figure 5(a) shows the crack located at $(x, y)=(50,0)(\mathrm{mm})$ in the shapedistorted billet. The length of the crack was set to be $3 \mathrm{~mm}$, and its direction was set at angles of $90^{\circ}$ with the $y$-axis. Figure 5(b) shows images obtained with the previously proposed method. The apparent sound velocity at point $(50,0)$ was estimated to be $5406 \mathrm{~m} \cdot \mathrm{s}^{-1}$, and that near the 


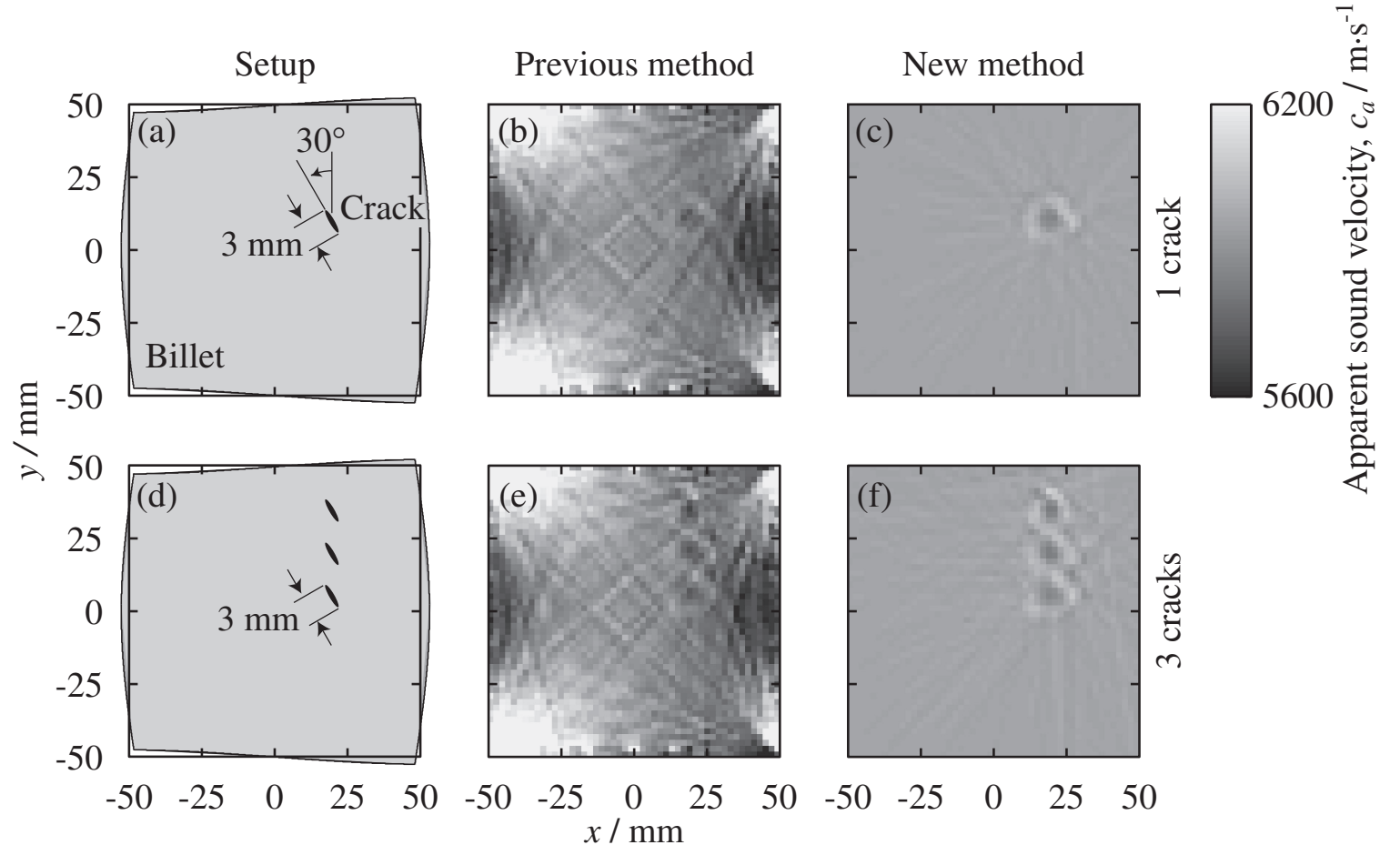

Fig. 4 Simulation setup and reconstructed images of apparent sound velocity: (a) setup of a single crack; (b) and (c) images obtained with previously proposed method and newly proposed method, respectively; (d) setup of three cracks; (e) and (f) images obtained with previously proposed method and newly proposed method, respectively.

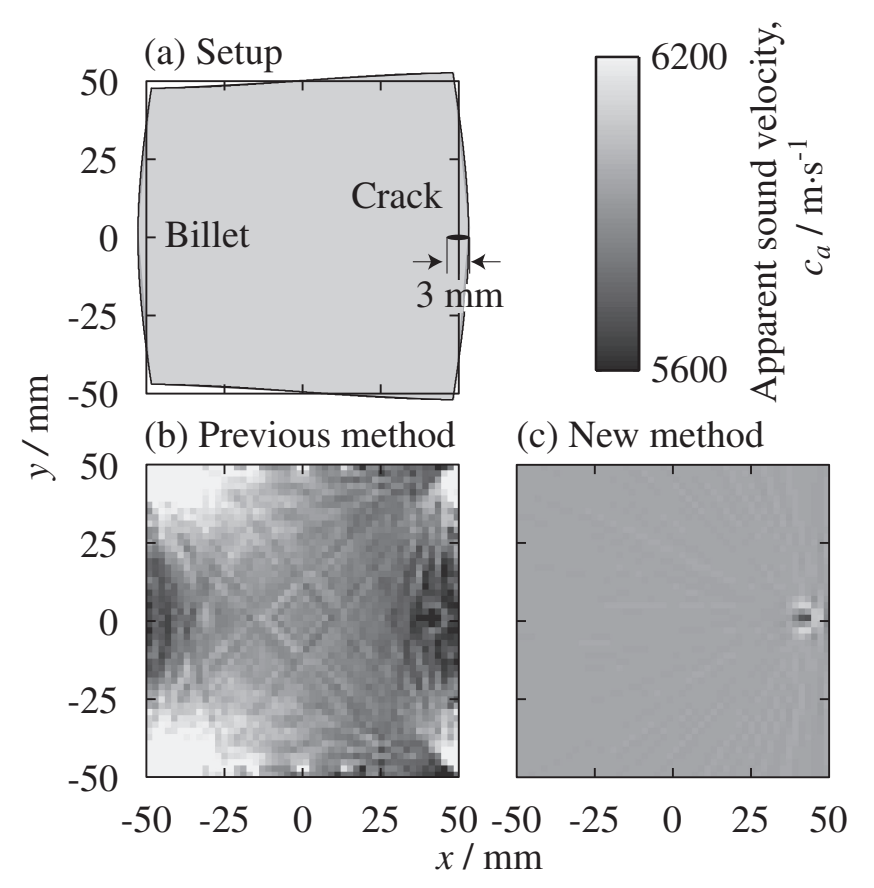

Fig. 5 Simulation setup and visualization of a single crack located near the billet surface: (a) simulation setup; (b) and (c) images obtained with previously proposed method and newly proposed method, respectively.

crack was approximately $5600 \mathrm{~m} \cdot \mathrm{s}^{-1}$. In other words, the apparent sound velocity at the crack is lower than that in the area around it; this is because the difference in TOF measured along the propagation path passing through the crack increased owing to both the diffraction of the longitudinal waves at the crack and the stretching of the propagation path because of shape distortions. However, it is difficult to distinguish between the decrease in the apparent sound velocity due to the existence of a crack and that due to shape distortions, because there is a much larger decrease in the apparent sound velocity due to shape distortions. Therefore, with the use of the previously proposed method, a crack near the surface cannot be detected. Figure 5(c) shows the image obtained with the newly proposed method. In this case, the crack can be identified even if it is located near the surface. This means that the performance of this method is not affected by the billet's distortion.

\section{Conclusion}

In this paper, we proposed the new method for the detection of cracks in cast billets, which combined ultrasonic CT with measurements of the TOF at multiple planes. The detection ability of this method was evaluated by numerical simulations of a shape-distorted billet. The longitudinal waves propagating through the billet were estimated by the TLM method. With the crack detection method that we had previously proposed, it was difficult to identify cracks because the decrease in apparent sound velocity was caused not only the crack but also the shape distortions. On the other hand, the newly proposed method prominently detected the cracks whose length was equal to the wavelength of the input signal. In addition, it is possible to detect the crack near the surface of the billet because this method can compensate for the effects of shape distortions whose dimensional change is smaller than $5 \%$. Therefore, the newly proposed method is expected to be particularly useful for the detection of cracks in shape-distorted billets. A thorough experimental verifica- 
tion of the new method's reliability has already been planned for manufactured cast billets. A reduction in the number of measurement points remains to be investigated in a future study. The number of measurement points on each side of the billet was fixed to 50, which was so large that the total measurement time was very long. Our ultimate aim is to reduce the number of measurement points and subsequently the measurement time, while maintaining the same detection ability.

\section{REFERENCES}

1) M. Shimura: Trans. JIM 19 (1978) 589-597.

2) H. Yamaguchi, N. Matsubara, K. Fujisawa and S. Matsumoto: Tetsu to Hagane 70 (1984) 1210-1215 [in Japanese].

3) M. Tokizawa and N. Takatsuji: Trans. JIM 29 (1988) 69-79.

4) K. Fujimoto, M. Shimura and S. Satoh: Mater. Trans. 43 (2002) 101104.

5) M. B. Karamis and F. Nair: Wear 265 (2008) 1741-1750.

6) S. H. Hsiang and S. L. Lin: Int. J. Mech. Sci. 43 (2001) 1155-1177.

7) L. H. You, J. H. Hu, Y. H. Shi and J. J. Zhang: J. Mater. Process. Technol. 150 (2004) 62-69.

8) H. Kakimoto, Y. Takashi, H. Takamori, T. Tanaka and Y. Imaida: Mater. Trans. 50 (2009) 1998-2004.
9) T. Jayakumar, P. Palanichamy and B. Raj: J. Nucl. Mater. 255 (1998) 243-249.

10) M. Abe, T. Fujioka and Y. Nagata: Acoust. Sci. Technol. 23 (2002) 308-312.

11) H. Mitsui, K. Mizutani and N. Wakatsuki: Jpn. J. Appl. Phys. 48 (2009) 07GD05_1-5.

12) J. R. Klauder, A. C. Price, S. Darlington and W. J. Albersheim: Bell Syst. Tech. J. 39 (1960) 745-808.

13) M. Pollakowski and H. Ermert: IEEE Trans. Ultrasonics 41 (1994) $655-659$.

14) I. Saito, K. Mizutani and N. Wakatsuki: Jpn. J. Appl. Phys. 46 (2007) 4537-4540.

15) H. Mitsui, A. Minamide, K. Mizutani and N. Wakatsuki: Collected Abstracts of the 2009 Autumn Meeting of Acoustical Society of Japan (2009) pp. 1285-1286.

16) K. Mizutani, K. Nishizaki, K. Nagai and K. Harakawa: Jpn. J. Appl. Phys. 36 (1997) 3176-3177.

17) N. Zhu, Y. Jiang and S. Kato: Energy 30 (2005) 509-522.

18) A. Minamide, K. Mizutani and N. Wakatsuki: Jpn. J. Appl. Phys. 48 (2009) 07GC02_1-8.

19) W. J. R. Hoefer: Alta Frequenza 58 (1989) 541-549.

20) L. Chai and Y. Kagawa: J. Sound Vib. 304 (2007) 587-605.

21) A. L. Daipra, M. D. Echeverria, A. Scandurra, O. Moncada and J. Sikora: Latin Am. Appl. Res. 35 (2005) 197-204.

22) H. Mitsui, K. Mizutani and N. Wakatsuki: Jpn. J. Appl. Phys. 49 (2010) 07HC13_1-6. 